\title{
Arsenic contents and associated health risk in soils of a mining area
}

Tepanosyan G. ${ }^{1}$, SAhakyan L. ${ }^{1}$, SAghatelyan A.

${ }^{1}$ The Center for Ecological-Noosphere Studies, NAS RA gevorg.tepanosyan@cens.am, lilit.sahakyan@cens.am, ecocentr@sci.am

Mining related activities alter the chemical composition of soils and have negative reflection on people's health. Among the toxic elements released into the environment through ore processing, dressing and smelting activities arsenic (As) is unique due to its ability to pose both noncarcinogenic and carcinogenic effects to human health. Therefore, As contents were determined using X-ray fluorescence spectroscopy (XRF Innov-X-5000 (USA)) in soils of Alaverdi city (Armenia) hosting the Alaverdi Copper Smelter (ACS) in order to evaluate As pollution levels and assess human health risk.

The obtained results showed that As contents range from $13-2038 \mathrm{mg} / \mathrm{kg}$ with the mean of $94 \mathrm{mg} / \mathrm{kg}$ which exceeded nationally stated maximum acceptable concentration (10 $\mathrm{mg} / \mathrm{kg}$ ) by 9.4 times. The spatial distribution of pollution levels showed that the hazardous level of pollution was mainly observed close to the major roads and near the ACS and its surroundings where mining waste and dumping sites, as well as Arsenic burial is located. Moreover, during the fieldwork it was identified that the local authorities are using the sludge of the ACS as an abrasive material to cover frozen roads during the wintertime. The allows to explain why high contents of As were also detected close to the major roads.

The non-carcinogenic health risk assessment was performed using the US EPA risk assessment method [1]. The results showed that the main residential part of the city was under the medium level of the risk whereas the areas close to the ACS were under the high level of non-carcinogenic risk. This suggested that an adverse health effect to children can be expected. Carcinogenic risk was above the allowable level of $10^{-6}$ in the entire city territory.

The limited information provided in the literature inferred that the results obtained within this study are realistic. Particularly, in the study area the indicators of case rate and mortality exceeded the average national indicators in Armenia by 2-3 times and the incidence of oncological diseases exceeds the standard occurrence with 10 times.

[1] Risk Assessment Information System, 2020. https://rais.ornl.gov/tools/rais_chemical_risk_guide.html (accessed 13.02.2020). 